\title{
Observations of High-Frequency Activity of EV Lacertae
}

\author{
B.E. Zhilyaev, I.A. Verlyuk \\ Main Astronomical Observatory, National Academy of Sciences of Ukraine, \\ $252650 \mathrm{Kiev}$, Ukraine
}

\section{Introduction}

The flare star EV Lac shows a wide variety of activity phenomena on a wide range of time scales. These are the well-known "classical" solar-like flares, the short-time flare events (Gershberg \& Petrov 1986, Karapetian \& Zalinian 1991, Tovmassian \& Zalinian 1988, Tsvetkov et al. 1986, Zhilyaev 1994), and the longduration variations caused by starspots and rotation (Petterson 1980). However, the "classical" flares are not such a frequent phenomenon as one gets used to think. As noted by Roizman \& Kabitchev (1985), a considerable fraction of flares shows a complicated multipeak structure with sudden onset and decay. Their interpretation along the lines of classical models favored for solar-like flares is very problematic.

Here, we report the detection of high-frequency chaotic variability (flickering) in EV Lac. Optical flickering is observed in cataclysmic binaries, and in X-rays it is observed in low-mass X-ray binaries. As a rule, flickering is an accretion phenomenon. Strange as it may seem, EV Lac as a visual binary has the analogous property.

\section{A test for detection and estimation of chaotic variability}

Denote the sampling values of the mean count rate and of the variance as $\bar{n}$ and $s^{2}$. The variance has the physical meaning of the power of fluctuations. It consists of three components caused by Poisson noise, scintillations and intrinsic stellar variability:

$$
s^{2}=s_{\mathrm{p}}^{2}+s_{\mathrm{sc}}^{2}+s_{*}^{2}
$$

The first term is equal to the mean count rate of star and background

$$
s_{\mathrm{p}}^{2}=\bar{n}=\bar{n}_{*}+\bar{n}_{\mathrm{b}}
$$

The second term evaluates a contribution of atmospheric scintillations to the total variance. It may be defined as

$$
s_{\mathrm{sc}}^{2}=\frac{1}{T} \int_{0}^{T}\left[f(t) n_{*}(t)\right]^{2} d t=\epsilon_{\mathrm{sc}} \bar{n}_{*}^{2}
$$


where $f(t)$ is the light wave envelope in the turbulent atmosphere. The quantity $\epsilon$ defined as

$$
\epsilon=\frac{s^{2}-\bar{n}}{\bar{n}_{*}^{2}}=\frac{s_{\mathrm{sc}}^{2}+s_{*}^{2}}{\bar{n}_{*}^{2}}=\epsilon_{\mathrm{sc}}+\epsilon_{*}
$$

specifies the power of scintillations in the operating frequency range $1 / T \div 1 / 2 \Delta t$, where $\Delta t$ is the sampling time. The quantity $\epsilon_{\mathrm{sc}}$ depends on atmospheric conditions as well as on the aperture of the telescope. It is independent of intensity $\bar{n}_{*}$ (Tatarsky 1967).

Using the standard procedure (Kendall \& Stuart 1946), the error variance for $\epsilon$ can be found

$$
D[\epsilon]=\frac{1}{\bar{n}^{4}}\left\{D\left[s^{2}\right]+\left(\frac{2 s^{2}}{\bar{n}}-1\right)^{2} D[\bar{n}]\right\}
$$

where $D$ is the variance operator. For variances of $\vec{n}$ and $s^{2}$ we have

$$
D[\bar{n}]=\frac{s^{2}}{N}, \quad D\left[s^{2}\right]=\frac{2 s^{4}}{N-1}
$$

where $N$ is the length of the measurement series.

The actual value of $\epsilon_{\mathrm{sc}}$ caused by scintillations can be determined from measurements of a reference star assuming $\epsilon_{*} \equiv 0$. To detect atmospheric scintillations, one can assume the null hypothesis: $s^{2}=\bar{n}$. In view of (5) a detection threshold can be set

$$
\epsilon_{\text {th }}=\alpha \sqrt{D[\epsilon]}=\frac{\alpha}{\bar{n}} \sqrt{\frac{2}{N}} .
$$

For a time series with normal density, $\alpha=1.96$ corresponds to a $95 \%$ confidence level. The quantity $\epsilon$ specifies an accuracy limit of stellar photometry in the operating frequency range at given atmospheric conditions. The above quoted expressions open the way for the detection of intrinsic chaotic fluctuations of stars masked by atmospheric noise.

\section{Observations and results}

High-frequency chaotic variabiliy was found in EV Lac in the $B$ band. Observations were obtained with a high-speed two-star photometer with $0^{8} 05$ integration time at the $60 \mathrm{~cm}$ telescope at the Mount Terskol Observing Station in North Caucasus (3100 m above sea level). Monitoring was carried out on 1991 October 13 , and on 1993 November 6, for about half an hour. Simultaneous measurements of a reference star were made in order to control and estimate atmospheric noise.

Our observations reveal that intrinsic flickering takes place in EV Lac. The results are presented as a power vs. time curve in Fig. 1. Each dot is the result of a 1.0 estimate of $\epsilon$. In addition, the curve has been smoothed by a movingaverage filter for the purpose of noise cancelling. Flickering in EV Lac shows a nonstationary quasiperiodical character on a time scale of $140 \mathrm{sec}$. The maximum power of the variable component is equal to about $5 \cdot 10^{-3}$ in the frequency band of $10 \mathrm{~Hz}$. At minimum it drops below the detection threshold equal to about 


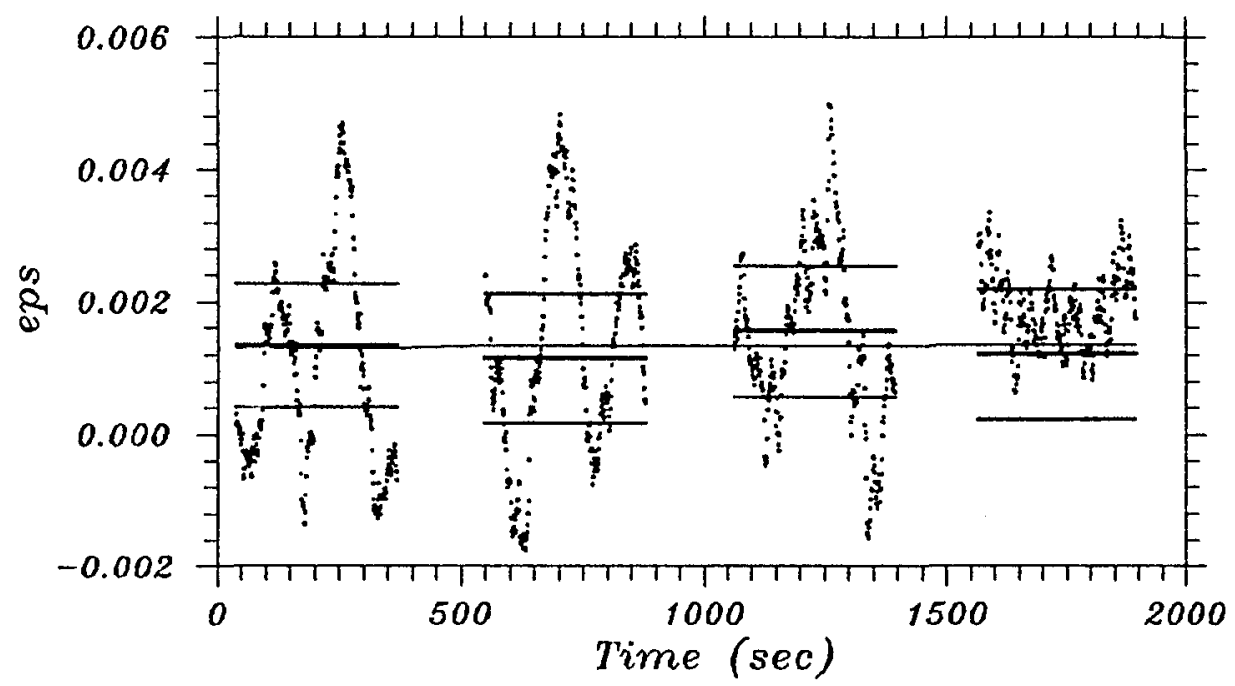

Fig. 1. EV Lac on 1991 Oct. 13. Variations in the power of fluctuations in the $B$ band. Each dot is a result of a $1.0 \mathrm{sec}$ estimate of $\epsilon$. The mean values and the error limits of $\epsilon$ are presented.

$4 \cdot 10^{-4}$. The mean power of the variable component remains constant during the observational run and equals $(9 \pm 2) \cdot 10^{-4}$.

The power of atmospheric scintiallations derived from the reference star data remains constant to an accuracy of $3 \%$ and equals $(322 \pm 9) \cdot 10^{-6}$. EV Lac demonstrates the same behaviour of the power curve in the second observing run on 1993 November 6 . Both runs were performed at fair atmospheric conditions with a low level of atmospheric scintillations.

Acknowledgements: The research described in this publication was made possible in part by Grant No. UCBOOO from the International Science Foundation.

\section{References}

Gershberg R.E., Petrov P.P., 1986, in Flare Stars and Related Objects, Publishing House of the Acad. of Sci., Yerevan, p. 38

Karapetian A., Zalinian V., 1991, IBVS 3550

Kendall A., Stuart A., 1946, The Advanced Theory of Statistics, Vol. 1, Charles Griffin \& Comp., London

Petterson R.B., 1980, AJ 85, 871

Roizman G.S., Kabichev G.I., 1985, AZh 62, 1095

Tatarsky V.I., 1967, Propagation of Waves in Turbulent Atmosphere, Moscow, Nauka

Tovmassian H, Zalinian V., 1988, Astrofiz. 28, 131

Tsvetkov M., Antov A., Tsvetkova K., 1986, Comm. Konkoly Obs. No. 86, 435

Zhilyaev B.E., 1994, Kinematics and Physics of Celestial Bodies 10, 32

Zhilyaev B.E., Verlyuk I.A., 1995, this volume, p. 80 